\title{
Surfaces
}

\section{L'OBLITÉRATION DU NOM. CONSIDÉRATIONS SUR LE ROMANESQUE AQUINIEN ET SUR LE SUJET-NATION QUÉBÉCOIS}

\section{Jacques Cardinal}

\section{Volume 3, 1993}

URI : https://id.erudit.org/iderudit/1065101ar

DOI : https://doi.org/10.7202/1065101ar

Aller au sommaire du numéro

Éditeur(s)

Les Presses de l’Université de Montréal

ISSN

1188-2492 (imprimé)

1200-5320 (numérique)

Découvrir la revue

Citer cet article

Cardinal, J. (1993). L'OBLITÉRATION DU NOM. CONSIDÉRATIONS SUR LE ROMANESQUE AQUINIEN ET SUR LE SUJET-NATION QUÉBÉCOIS. Surfaces, 3. https://doi.org/10.7202/1065101ar

\section{Résumé de l'article}

Sur la base d'une théorie de la nomination et de la signature comme ressorts symboliques de l'inscription du sujet dans l'histoire, l'auteur analyse le statut parfois ambigu du sujet québécois et montre comment ce celui-ci est représenté dans les oeuvres de l'écrivain Hubert Aquin.

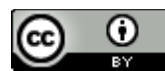

Ce document est protégé par la loi sur le droit d'auteur. L'utilisation des services d'Érudit (y compris la reproduction) est assujettie à sa politique d'utilisation que vous pouvez consulter en ligne.

https://apropos.erudit.org/fr/usagers/politique-dutilisation/ 


\title{
L'OBLITÉRATION DU NOM
}

CONSIDÉRATIONS SUR LE ROMANESQUE AQUINIEN ET SUR LE SUJET-NATION QUÉBÉCOIS

Jacques Cardinal

RÉSUMÉ

Sur la base d'une théorie de la nomination et de la signature comme ressorts symboliques de l'inscription du sujet dans l'histoire, l'auteur analyse le statut parfois ambigu du sujet québécois et montre comment ce celui-ci est représenté dans les oeuvres de l'écrivain Hubert Aquin.

\begin{abstract}
On the basis of a theory of naming and signing as springboard for the inscription of the subject in history, the author analyses, namely in $H$. Aquin's work, the at times ambiguous status of the québécois subject.
\end{abstract}

Je dois maintenant à la fois être et ne pas être.

\section{S. Kierkegaard}


La problématique de la représentation du Sujet-Nation québécois sur la scène de l'histoire ne se laisse peut-être pas penser sans une réflexion sur les conditions de possibilité de la lisibilité du nom du signataire de la loi dans le régime politico-symbolique propre à l'État constitutionnel moderne, et de l'économie de la lisibilité de la lettre ou du nom propre en général.

À cet égard, on se rappellera que la lisibilité de la lettre à l'oeuvre dans notre culture s'inscrit dans un procès selon lequel le nom (l'écrit, le graphein) est toujours déjà oblitéré par la voix (la phonè) en laquelle se recueille, pour le sujet, l'expérience de la présence du présent et de la vérité (Derrida 1967). Il résulte de cela que le protocole de lecture et d'écriture du nom, et de la loi comme reconnaissance de ce nom sur la scène de l'histoire, est inscrit, dès l'origine, dans une coupure où le nom est la re-marque d'un nom originairement perdu ou inaccessible, mais retrouvé, après-coup, dans le corps (soma) mortifère de la lettre ou du nom. Ce nom suture ainsi, dans le fantasme de cette métaphysique de la présence, cette différence originaire qui est déposée dans le nom comme nom de la loi ou de la fondation. Cette différence originaire, le nom (de la loi, de la fondation) la suture dans une lettre que l'on peut dire monumentale ou funéraire, dans la mesure où elle est comme le recouvrement d'une origine qui est, en même temps, présente et absente dans ce nom. La lettre est ainsi une stèle sur laquelle le nom de la loi se donne à lire selon un double mode où il est en même temps reconnu comme le nom de la loi et, cependant, interdit quant à la possibilité de le dévoiler et de le maîtriser comme nom de l'origine[1]. Le nom relève aussi d'une double structure puisqu'il marque l'ancrage du sujet dans le symbolique, et s'inscrit ensuite imaginairement dans la petite histoire du sujet (Major 1984).

Cette économie de la lettre, la philosophie de la déconstruction (pour la nommer ainsi que la nomme une certaine doxa philosophique) ainsi qu'un certain discours psychanalytique, en ont pris la mesure afin d'y reconnaître, dans le contexte plus général de la modernité philosophique et politique où le sujet décline désormais son nom sur le mode de la volonté et de l'autonomie, l'illusion d'une transparence absolue (dé-liée, donc) quant à la lisibilité du nom comme fondation de l'État-Nation moderne (Derrida 1984). En cela, cette fondation moderne du politique à travers l'État a pu céder au désir de l'origine (ou du Savoir Absolu hégélien) comme présence à soi du sujet et de la vérité dans l'histoire. Le nom de la fondation moderne était encore, dans ce cas, arraisonné à un autre nom (ou Idée), parfois sur le mode de la dénégation, et ne faisait qu'accomplir le destin de la métaphysique occidentale. L'histoire de la fondation politique moderne, et à moins de céder au fantasme du nom de l'élection nationale, est ainsi inscrite dans le nom comme lieu de la différence originaire, c'est-à-dire dans le nom comme manque et comme perte du nom infini de Dieu, par exemple, et comme condition de possibilité de l'histoire (Major 1986). C'est dire enfin que le protocole d'écriture et de lecture de la signature constitutionnelle moderne n'échappe pas à cette détermination, et que s'il y a un mot d'ordre inhérent à ce savoir, c'est sans doute celui de ne pas céder au nom comme 
captation de l'origine, là où le sujet reconnaît la finitude de son nom sur la scène de l'histoire. En cela, sans doute, le discours sur l'identité du sujet (national) peut-il changer dans la mesure où ce sujet reconnaît le procès de cette méprise originaire du nom.

Cela dit, et il convient d'y insister, le discours de la reconnaissance de la fondation comme différence originaire n'abolit pas comme tel le concept d'identité du sujet pour autant que cette identité se donne à lire dans un nom propre. Il ne fait que reconnaître ce nom comme originairement oblitéré, et peut dès lors le lire dans cette coupure où l'identité du sujet se noue à l'histoire par la finitude d'un certain désir. En cela, le sujet est toujours déjà le sujet d'un nom propre et s'inscrit dans la loi au nom de ce désir qui le distingue ou le rend discernable.[2] Ainsi ce n'est pas le discours de la subjectivité moderne comme maîtrise radicale de l'expérience cognitive qui s'affirme, comme le voulait Descartes, par exemple, mais un sujet dont le propre est d'être travaillé par son désir. Par conséquent, cette critique "déconstructionniste" du concept d'identité ne porte, en définitive, que sur l'inscription du sujet dans la métaphysique ancienne et moderne. Cela ne l'empêche donc pas de reconnaître par ailleurs qu'il y a aussi un sujet dont l'identité est déposée dans la discernabilité de son nom et de son désir. Or, c'est ce désir du sujet, en l'occurrence le désir du Sujet-Nation québécois, qu'il est sans doute intéressant d'analyser à l'heure où l'on condamne souvent ce désir de reconnaissance au nom, ambigu à mon avis, de cette critique de la métaphysique du sujet. Il va de soi ici que je n'adhère en rien au nationalisme romantique et à la métaphysique de l'enracinement et du terroir qu'il implique. Je voudrais plutôt penser l'économie de la loi du nom telle que peut-être nous la désirons au fil de l'histoire comme mise en scène d'un désir.

En cela, on peut reconnaître que le nom du Québec est un nom propre et qu'il porte, ce faisant, sa propre histoire comme sédimentation d'un désir. Dans ce cas, le nom du sujet individuel québécois se noue au nom du Québec comme mémoire, et tel qu'en lui-même cependant l'éternité peut le changer. Heureusement. C'est pourquoi il m'importe de réfléchir sur la complexité du noeud qui lie ensemble le nom du sujet individuel québécois avec celui du Québec. Bien entendu, ce noeud n'est pas lisible dans une seule figure topologique puisqu'il s'articule, chaque fois, sur la petite histoire du sujet et selon son propre travail d'élaboration fantasmatique. Mais le nom du Québec est aussi un nom qui se donne à lire dans la loi du nom comme désir, c'est-à-dire dans le protocole de lecture et d'écriture de la discernabilité. Or, la discernabilité du nom n'est pas celle de l'inaltérabilité d'un nom qui se désirerait, jusqu'au délire, sur le mode de la pureté généalogique; elle n'est, en fait, qu'un nom qui s'ouvre à la reconnaissance de son histoire sur le mode de la fondation comme coupure et oubli. La discernabilité du nom n'est, en définitive, que le nom que nous offrons à l'autre comme désir. Et c'est en vertu de cette discernabilité que le nom du sujet donne prise, en le jouant aussi comme indécidable quant à l'ultime nom du désir, à cette rencontre avec le nom de l'autre également discernable. C'est donc à partir de cette discernabilité toujours déjà 
imaginaire du nom propre que la reconnaissance d'un sujet politique peut enfin se donner à lire dans cette double marque de l'identité et de la différence.[3]

Quel est donc, dans cette perspective, le statut du nom du Québec? On pourrait avancer que le nom du Québec est un nom (doublement) oblitéré sur la scène de l'histoire. Il en serait ainsi en raison du statut de sa signature constitutionnelle qui est, dans une certaine mesure, arraisonnée à la signature du maître colonial anglo-saxon. En cela, le nom du Québec serait un nom doublement oblitéré dans un régime constitutionnel qui est celui, non pas d'une double signature, mais d'une contresignature. Il en serait ainsi car cette signature est, selon le discours d'une certaine historiographie, le résultat tout à fait ponctuel d'un compromis politique. Sans entrer dans toutes les péripéties de cet acte de fondation, on se rappellera en effet que les Pères de la Confédération canadienne, à l'initiative de John A. McDonald et de Georges E. Cartier, ont ratifié l'A.A. N.B. dans le but notamment de se prémunir contre une possible invasion américaine. Pour les royalistes du Haut-Canada (l'Ontario) et pour le BasCanada d'alors (le Québec), cette alliance était plus que raisonnable, et n'a d'ailleurs été possible que sur la base libérale de la démocratie parlementaire. Mais le poids politique (le bargaining power) de la signature constitutionnelle du Québec dans le régime fédéral a toujours été problématique. On se rapellera à cet égard l'épisode de la conscription où, malgré la promesse de McKenzie King, le Bill 80 a été voté en 1942, suite à un plébiciste, pour libérer le gouvernement de sa promesse de nonconscription, laquelle visait surtout le Québec. Or, le Québec, qui avait voté "non" dans une proportion de 71,2\% lors de ce plébiciste, a été finalement forcé de s'aligner sur l'autre signature et ce, même si cette conscription ne fut imposée que deux ans plus tard (et pour un nombre déterminé de soldats $(16,000))$ afin de tempérer les conflits et de protéger l'unité canadienne (Bilodeau 564). Demi-mesure qui reflète bien, en somme, les compromissions souvent douloureuses du fédéralisme canadien. Ainsi, et malgré le rapport de force démocratique qui a pu infléchir cette politique, celle-ci a tout de même finalement été appliquée. C'est donc une logique politique basée sur la reconnaissance d'une majorité sans discernement qui a finalement prévalue au détriment des droits légitimes d'une minorité. Il est vrai cependant qu'à l'époque on ne parlait pas encore des "deux peuples fondateurs" (sans oublier les Amérindiens...), et encore moins de multiculturalisme. Cet épisode politique montre donc l'ambiguité de la reconnaissance du Québec dans la constitution canadienne. Cela est d'autant plus vrai qu'en ce qui concerne cette question, les Canadiensfrançais savaient le peu d'influence qu'ils exerçaient au sein de l'armée canadienne. Le poids politique de la signature du Québec s'est donc avéré, en cette occasion décisive, plutôt faible. Et c'est le statut de cette signature qui fait l'objet depuis ce temps d'un incessant débat politique.

Ce n'est évidemment pas le fédéralisme en tant que mode d'organisation politique qui est en cause ici, mais le mode de reconnaissance symbolique des signataires de la constitution. Or, dans ce cas, on peut affirmer que la 
valeur de la signature du Québec sur la scène constitutionnelle canadienne, et de l'histoire comme mise en oeuvre du droit des peuples à disposer d'euxmêmes, semble prise dans le noeud d'une certaine ambiguité. Cette signature est en effet ambiguè parce qu'elle est à la fois reconnue (en tant que le nom d'un sujet mis en position de Sujet-Nation sur la scène de l'histoire se donne à lire sur un texte légal, c'est-à-dire reconnu par la communauté du droit international), et cependant méconnue dans la mesure où cette signature est arraisonnée par la contresignature du maître colonial. À la fois reconnu et méconnu, le Sujet-Nation québécois ne serait donc pas à proprement parler un "colonisé", mais plutôt un "demi-colonisé" comme le disais déjà Jacques Ferron (173). Cet état de demi-colonialisme me semble du reste assez singulier et explique peut-être la difficulté de dénouer la sempiternelle question de la souveraineté nationale que nous avons ou croyons avoir sur une scène et n'avons pas ou croyons ne pas avoir sur une autre.

Il faut préciser, toutefois, que depuis le rapatriement constitutionnel de 1982 , et suite aux péripéties de la négociation où le Québec a finalement été isolé, le gouvernement de René Lévesque a refusé de signer ce texte constitutionnel. Cette signature est donc illisible depuis lors, mais cela a peut-être permis d'entrer dans une autre phase de négociation où, suite aux Etats généraux de la Commission Bélanger-Campeau sur l'avenir constitutionnel du Québec et du Rapport Allaire, la reconnaissance du Québec comme société distincte, sinon comme État-Nation, est posée explicitement dans la performativité politique et juridique de cette signature constitutionnelle. Le régime de la double oblitération du nom demeure, mais il s'est aussi dénoué quelque peu dans la mesure où le gouvernement de Robert Bourassa fait de cette signature, et de ce qu'elle représente politiquement, au moins jusqu'à maintenant, l'enjeu de la reconnaissance du nom du sujet québécois sur la scène politique canadienne. En cela, le débat politique me semble posé à la bonne place, car cette signature constitutionnelle serait enfin reconnue comme l'expression de l'autonomie du Québec, et ce même dans le cas où le Québec demeurerait dans la confédération. Mais C'était avant que R. Bourassa, faisant fi du Rapport Allaire, ne prenne l'initiative de négociations qui ont conduit à l'entente de Charlottetown qui fut finalement rejetée lors du référendum de l'automne 1992.

Cela dit, cette structure symbolique du sujet québécois semble également se compliquer du fait que le sujet canadien se perçoit aussi comme un colonisé, à l'égard des États-Unis cette fois (Grant 1969). Sujet colonisé par un sujet colonisé, le sujet québécois serait ainsi noué à son désir de reconnaissance non seulement selon une double oblitération, mais aussi, peut-être, par le savoir de la faillibilité de son maître. Ce qui évidemment ne l'empêche pas, bien au contraire, d'en désirer un parfois qui soit parfaitement infaillible. 
Il résulte de ce régime de la contresignature et du nom (doublement) oblitéré, une économie assez singulière de la représentation du nom de la loi au Québec que le texte aquinien semble porter de façon tout à fait symptomatique en ce qu'il se donne à lire dans ce que l'on pourrait nommer la double imposture du nom anamorphosé (illisible ou indiscernable) et du désir fantasmatique de la fondation originaire tel qu'il voudrait s'incarner dans le fétichisme du grand texte national. Cette double imposture désigne dans ce cas le procès par lequel le sujet serait noué à son nom, et à son désir, sur le mode de l'illisibilité et de la méprise. Im-posture, donc, dans la mesure où le nom du sujet n'est pas posé dans le protocole de la reconnaissance symbolique et constitutionnelle; mais, imposture aussi, dans le sens où ce sujet se joue la scène hystérique de l'imposteur qui se cache derrière la litanie des noms qui ne satisfait jamais sa demande de nomination et d'identité. À cet égard, je voudrais analyser d'abord l'impasse transférentielle à l'oeuvre dans Prochain Épisode.

On le sait, Prochain Épisode met en scène une confrontation entre le narrateur révolutionnaire et le maître de la loi visant à déterminer à qui revient la maîtrise narrative des actions du sujet dans le cours de l'histoire. [4] Or, cette confrontation échoue chaque fois que, arme en main devant le maître de la loi, le narrateur est soudainement "frappé de stupeur cataleptique" et ne parvient donc pas à commettre le geste homicide capable de le libérer de cette emprise. Cette catalepsie, en effet, le laisse interdit devant cet ennemi qui, tout à la fois, le fascine, le ravit, et l'inquiète étrangement. S'il en est ainsi, c'est sans doute que le narrateur suppose son ennemi omniscient. En cela, et malgré toutes les ruses qu'il met en oeuvre, il ne peut le déjouer car l'autre sait toujours déjà ce qu'il pense et jette ainsi le doute dans la conscience du narrateur quant à la maîtrise de ses gestes. [5] Cette confrontation est donc, en définitive, condamnée à l'échec puisque le narrateur est, d'entrée de jeu, arraissonné au jeu du maître qui est le maître du jeu. Cette impasse, on pourrait dire qu'elle est celle d'une captation imaginaire au champ de l'Autre (avec un grand A) dans la mesure où, dans ce cas, le sujet fantasme son rapport à l'autre (avec un petit a), c'est-à-dire en tant que sujet voué à la finitude, sur le mode symbolique du nom infini de la vérité et de la fondation originaire (Lacan 53). Or, ce nom infini de la loi est, dans le texte, à la fois lisible et illisible, et se donne au narrateur sur le mode d'un nom impossible à dénouer, à maîtriser. Telle est, semble-t-il, la structure de ce transfert où le narrateur de Prochain Épisode pense son rapport à l'autre sur le mode d'une captation imaginaire au champ de l'Autre, c'est-à-dire au symbolique. Cette capture n'est cependant pas totale, car dans ce cas le sujet serait enfermé dans la psychose. Le romanesque aquinien donne plutôt à lire cette capture comme un état limite (borderline) en laquelle le sujet, alternativement, se possède et ne se possède plus. Il ne joue peut-être cette scène de la possession que pour éprouver la solidité du lien qui le détermine. Cet état limite ne serait ainsi que l'indécision propre à ce ratage symbolique inhérent à cette structure de la double oblitération de son nom. 
Sur ce point, la question que l'on peut dire quasi épidémique de l'imitation au Québec me paraît symptomatique de cette maladie. L'on sait qu'en ce domaine on peut se targuer d'avoir le champion en André-Philippe Gagnon, le ventriloque aux mille et une voix et personnages. Faut-il reconnaître dans cette compulsion à l'imitation, ce désir de l'autre comme captation imaginaire? Il est vrai, cependant, que cette mise en scène peut être parfois ironique, et permettre en cela au sujet d'en décoller quelque peu. Mais ce n'est pas toujours le cas. Quoiqu'il en soit, c'est généralement dans la virtuosité de la performance, c'est-à-dire dans la capacité qu'a le sujet de coller à son sujet que l'on reconnaît la réussite du fantasme. Ce fantasme serait comme la mise en acte d'un transfert massif sur l'autre comme lieu de la vérité du désir du sujet. Le cas de Will James, alias Ernest Dufault est sans doute le plus pathétique (Larose 1989). Certes, on peut arguer que "Je est un autre" et qu'il est salutaire de se reconnaître "étranger à soi-même". Mais la légitimité de cette expérience n'est-elle pas possible aussi que pour un sujet qui est déjà dans une économie du nom en laquelle se remarque la coupure imaginaire avec le nom originaire de la fondation? À cause de cela, cette expérience n'est peut-être, pour le sujet québécois, que le lieu d'une imposture hystérique puisqu'elle ne s'élabore pas sur un désir qui serait celui de la coupure, mais plutôt dans celle de cette captation imaginaire au champ de l'Autre. Ce "paradoxe du comédien" ne serait donc pas nécessairement ici celui d'un "sujet sans qualités" qui se donnerait encore de la maîtrise à ce jeu de l'imitation dans la poïesis, mais le lieu d'une certaine imposture quant au lien transférentiel avec l'autre (LacoueLabarthe 15-35). Mais revenons à Prochain Épisode afin d'y lire, peut-être, une certaine économie du nom.

L'impossibilité d'en découdre avec cette captation est d'ailleurs lisible dans ce fatras de signatures illisibles et cryptiques qui trame le fil de la narration de Prochain Épisode.[6] En effet, le nom par qui la loi arrive se refuse, pour le narrateur, à sa lisibilité pour ainsi dire publique, et donc à la finitude de sa profération, comme le nom interdit d'une loi dont il ne maîtrise pas l'économie. Dans ce cas, le nom de la loi s'inscrit dans le texte comme le nom /pp. 16-17/ anamorphosé d'une reconnaissance en souffrance du nom par lequel le sujet tient au fil d'une histoire dont il peut se dire le signataire. À cet égard, et sans entrer dans toutes les circonvolutions de cette capture, on peut dire que le romanesque de Prochain Épisode met aussi en scène le ratage symbolique de cette inscription du nom du narrateur qui porte le désir de cette reconnaissance du nom du Québec dans le cours de l'histoire. [7] Ce ratage symbolique se donne à lire ici dans l'illisibilité de la métaphore paternelle, du Nom-du-Père (Lacan 278) comme instauratrice de la filiation et du désir, et la défaite des Plaines d'Abraham s'inscrit dans le texte comme la scène originaire de ce ratage. Le corps du père jonche ainsi le sol de la représentation historique excluant le sujet du procès symbolique par lequel se noue, dans la coupure, la fondation originaire et l'élaboration de la filiation historique. Ce serait ce legs du nom du père qui, comme une peste, rendrait indiscernable le nom du sujet québécois. En cela, on peut y lire aussi toute une rhétorique de la parataxe et de la mélancolie morbide comme mise en oeuvre d'un romanesque de l'échec et de la possession, mais 
promis, cependant, après la violence révolutionnaire et cathartique, au grand dénouement historique.[8] L'imposture, donc, d'un nom anamorphosé dans le tissu de la représentation constitutionnelle, et qui ne se donnerait à lire que sous l'angle d'un certain retour du refoulé.

Quant au fantasme de la fondation originaire, le romanesque de Trou de mémoire le met en scène dans une rhétorique néo-baroque où, malgré le régime de la polyphonie narrative et de l'imposture qui semble généralisée, le nom de la filiation se trouve ultimement arraisonné, en passant par l'économie fantasmatique de la violence sexuelle et meurtrière, au désir d'une femme qui assume, en le donnant à son enfant, le nom de ce père violeur et meurtrier: Magnant.[9] En cela, et si Prochain Épisode se donne à lire comme le roman de l'impasse historico-narrative, c'est-à-dire comme impossibilité d'actualiser la violence révolutionnaire sur la scène de l'histoire, on peut dire que Trou de mémoire se présente comme le passage à l'acte de cette violence prétendument fondatrice. Et c'est, ultimement, sous l'égide du désir d'une femme, de la mère, que l'acte de fondation du nom du père est pour ainsi dire sauvé de sa défaillance. Ce serait donc la mère qui occuperait, voilée dans sa toute puissance, le fond de la scène de ce théâtre des fondations.

La mise en scène de cette violence fondatrice, meurtrière et sexuelle, se donne à lire dans un double geste de monstration et d'effacement dont la figure de l'anamorphose donne la clé[10]. En effet, et si le texte semble à première vue indécidable quant à sa fin et à sa signification en raison de sa polyphonie, il s'inscrit cependant dans le désir du canon de la représentation classique comme protocole de la reconnaissance souveraine du sujet sur la scène de l'histoire; l'obscénité de cette violence fondatrice est ainsi anamorphosée dans le tissu de cette représentation mimétique qui la recouvre.[11] En d'autres mots, le procès de la lisisibilité du nom du sujet dans l'histoire est arraisonné à la poétique du discernable mimétique, et le récit de la violence fondatrice est l'obscénité incontournable sur laquelle celle-ci se fonde. Ce que donne à voir le romanesque de Trou de mémoire c'est donc, d'une part, cette déchirure dans le tissu de la représentation de l'histoire nationale du Québec et, d'autre part, la suture ou le cérémonial de lustration de la violence fondatrice qui sera anamorphosée dans le tissu de cette représentation. Ce n'est donc pas, en définitive, l'euphorie généralisée d'un sujet qui se joue des formes et de l'histoire, mais la dysphorie d'une écriture qui cherche à se réciter dans le phrasé linéaire et harmonieux de la loi du nom telle qu'elle peut être ici fantasmée comme inscrite dans la poétique d'un certain classicisme.

Cette violence fondatrice semble enfin résulter d'une pensée plutôt délirante quant au désir du sujet de se donner la loi sur le mode de la fondation originaire, $c^{\prime}$ est-à-dire sur le mode, présentifiable dans le présent, du symbolique. Sans entrer ici non plus dans toutes les péripéties de ce rituel de violence fondatrice, il faut souligner que ce rituel n'est ici possible que 
sur la toile de fond du discours hégélien de la dialectique du maître et de l'esclave, et tel que ce discours a pu être traduit dans la vision anthropologique d'Alexandre Kojève et dans la vision décolonisatrice de Frantz Fanon, où la lutte à mort constitue le moment incontour-nable de la reconnaissance du sujet.[12] Pris dans ce discours, lequel méconnaît aussi la force dialogique de la parole, le rituel de cette violence fondatrice apparaît comme une catharsis ou comme un cérémonial de lustration capable de libérer la Cité de la souillure, de la peste.

Ce discours est aussi inscrit, formellement, dans le discours esthéticométaphysique, hérité notamment de Nietzsche, Wöllflin et Eugenio D'ors, selon lequel l'histoire est lisible dans ce balancement où, alternativement, les formes incertaines, polyphoniques et spiraloïdes, sinon dionysiaques du baroque succèdent aux formes de la rationalité classique et apollinienne, dont le paradigme est le nombre d'or.[13] En cela, on peut dire que le baroque aquinien est un baroque malheureux dans la mesure où le sujet colonisé y reconnaît la condition symbolique de son désordre sur la scène de l'histoire ("Écrire en spirale comme un colonisé" comme le dit P.X. Magnant), avec l'espoir, cependant, d'ordonner cette parataxe dans le phrasé discernable d'une poétique classique. Mais le Romanesque aquinien semble plutôt se jouer de ces oppositions que de les poser comme une vérité de l'histoire.

Le texte aquinien semble ainsi dépositaire d'un double symptôme quant au protocole d'écriture et de lecture de la loi que l'on pourrait résumer dans la formule suivante: le désir de la loi s'y donne à lire dans le ratage symbolique de l'imposture du nom (doublement) oblitéré et du fantasme du texte national comme fondation originaire. C'est l'incidence de ce double symptôme qu'il faut peut-être analyser à l'heure où, sur la scène politique d'un certain libéralisme postmoderne, on se donne le mot d'ordre de la dialectique négative, de la différence, du différend, du pluralisme, de l'impureté, de l'ironie et de la parodie contre le récit totalisateur des Philosophies de l'Histoire moderne, ou de l'utopie d'une nouvelle fondation basée sur une pragmatique transcendantale, dialogique et contrefactuelle de l'espace publique (Habermas 348). Il s'agirait donc de poser la question du symptôme du texte aquinien sur cette scène polémique afin d'y penser, peut-être, l'occurrence du discours de la loi au Québec.

Comment donc penser ce double symptôme? On peut dire d'abord que la mélancolie du texte aquinien semble inscrite dans la douleur de cette (double) oblitération du nom, et qu'elle /pp. 22-23/ le porte pour ainsi dire jusqu'au délire dans le procès hyperbolique de son désir qui va de l'illisibilité (ou de l'indiscernabilité) jusqu'au fantasme de la transparence abolue du nom de la fondation comme origine. Il semble que cette mélancolie laisse apparaître pertinemment le refoulé de la condition coloniale, mais que son mode de sublimation (romanesque) est délirant en ce qu'il se donne à lire comme une capture imaginaire au champ de l'Autre. Dans ce cas, en effet, le 
sujet ne parvient pas à distinguer complètement l'imaginaire du symbolique, c'est-à-dire qu'il ne parvient pas à reconnaître que le symbolique est aussi imaginaire (Larose 1981, 1987). Cette disposition du sujet quant à l'économie de la représentation de la loi est peut-être caractéristique d'un certain désir du nom et de la loi au Québec. Or, qu'en est-il de cette disposition du sujet dans un contexte politico-idéologique que l'on nomme "post-moderne"?

Si la culture postmoderne peut être caractérisée comme étant celle de l'indétermination, de la fragmentation, de la décanonisation, de l'effacement du moi, de l'ironie, de l'irreprésentable, de l'hybridisation, de la carnavalisation, de la performance, du constructionisme et de l'immanence radicale, c'est aussi qu'elle se présente comme une nouvelle écriture de l'histoire et des valeurs qui étaient véhiculées par l'humanisme universaliste de la rationalité des Lumières (Hassan 1986). Cette réécriture serait donc le procès d'une certaine historiographie, et comme toute historiographie elle mettrait en jeu la question protocolaire des conditions de possibilité de la représentation et de la reconnaissance du sujet, qu'il soit national ou non. Elle remet également en question, il va de soi, la question de la pertinence de la représentation de l'histoire en tant qu'elle peut être dite celle d'un Sujet-Nation. Mais cette pertinence n'invalide pas la légitimité de la reconnaissance du nom ou d'une quelconque finitude comme avènement d'un désir sur la scène de l'histoire. Elle ne fait que la dénoncer, à bon droit, comme une disposition inhérente à la métaphysique occidentale et au nom de laquelle le sujet cède au désir de la présence. Mais le désir du SujetNation n'est pas nécessairement inscrit dans cette structure.

L'historiographie postmoderne remet donc en question pertinemment la métaphysique de la présence et du Livre comme totalisation du sens de l'histoire. En cela, elle se détache de la modernité pour se faire postmoderne. Mais l'économie discursive de la présence ne peut être confondue avec celle de la discernabilité. On pourrait donc arguer ici d'une complexion particulière quant au procès de cette historiographie postmoderne lorsqu'elle s'inscrit dans cette structure du nom (doublement) oblitéré du sujet québécois.

On peut reconnaître la légitimité de ce libéralisme postmoderne qui cherche à penser le Sujet-Nation sur le mode de la pluralité des centres afin de déplacer le centre de gravité de cette métaphysique du sujet moderne comme processus de fondation et d'exclusion. En cela, on peut y reconnaître également la légitimité d'une pensée qui cherche à penser l'autre comme différence. Mais la question demeure ouverte quant aux modalités politicosymboliques de cette reconnaissance. À cet égard, la légitimité de ce libéralisme postmoderne n'invalide pas la nécessité de cette économie de la loi et du nom qui, pour être lisible sur la scène de l'histoire, en appelle à une signature. La question reste cependant de savoir ce qui est fantasmatiquement investi dans cette signature. Si cette signature se donne d'abord comme le nom originaire de la fondation, elle peut être dénoncée comme un avatar de la métaphysique du sujet moderne; cependant, si elle se donne à lire dans la finitude du désir de son nom à l'égard d'un autre nom 
également fini, elle se noue enfin à elle-même sur le mode de la coupure imaginaire et symbolique, et peut dès lors élaborer son désir autrement que sur le mode de cette capture imaginaire au champ de l'Autre qui se traduit trop souvent par la haine (la paranoïa) ou l'amour (l'hystérie) absolus de l'autre. Dans ce cas, enfin, cette signature ne se donne à lire que dans l'événement et le contexte de son désir.

Il ne faut peut-être pas faire le deuil de cette signature constitutionnelle au nom de cette critique de la métaphysique du sujet dans la mesure où, dans ce cas, l'on méconnaît que le nom, la loi du nom, c'est d'abord celle de son désir. C'est dire en cela que ce nom ne se donne à lire pour un autre sujet, un autre nom, que dans le protocole de cette coupure où le nom ne se tient à sa place qu'à se tenir dans l'oblitération du nom infini qu'il n'est pas. C'est en cela seulement qu'il peut s'élaborer comme désirable et s'ouvrir à la différence. La légitimité, s'il en est une, de cette signature constitutionnelle du Québec apparaît donc, en définitive, comme le dénouement de cette (double) oblitération et de cette double imposture du nom anamorphosé. Mais elle ne le dénoue, enfin, que pour le nouer à la loi du nom et de son oblitération. Or, pour qu'elle puisse se dénouer, il faut aussi que ce sujet assume son nom comme désirable et qu'il assume le nom de sa langue comme désirable.

Cette langue, qui est en fait le désir du nom ou de cette signature est trop souvent l'objet d'une méprise. Le sujet québécois s'y méprend peut-être en effet quant à son désir, dans la mesure où il se donne souvent cette langue sur le mode du mauvais objet. En cela, il semble qu'il ne fait que se nommer, et malgré les dénégations et les sublimations, comme indésirable. Or, ce désir de l'indésirabilité n'est peut-être que le symptôme de son nom illisible qui ne s'est pas élaboré comme désir à l'oeuvre dans la loi du nom. D'autre part, force est de constater que cette langue n'est pas toujours désirée par l'autre. On peut penser ici à la situation de la reconnaissance de cette langue dans plusieurs écoles de Montréal, et non pas à l'intellectuel libéral postmoderne pour qui la chose mérite d'être entendue. En effet, et si c'est bel et bien le français qui est la langue d'enseignement dans ces écoles, on constate également que c'est l'anglais qui très souvent se donne comme la langue du consensus. L'anglais est désiré; le français, règle générale, ne l'est pas. Mais comment l'autre peut-il désirer ce qui ne s'est peut-être pas encore élaboré comme assomption d'un désir? L'incidence de cette méprise de la langue du sujet québécois serait peut-être celle aussi de son nom indiscernable sous le regard de l'autre.

L'État-Nation est encore, dans le contexte de notre modernité politique, et malgré sa crise de légitimation que le discours critique du postmodernisme lui fait subir avec raison sur certains aspects, le lieu d'inscription dialogique et démocratique de cette reconnaissance du Sujet-Nation. Le désir de cette signature n'est donc dicible aussi que dans ce protocole par lequel un nom se tient dans la chaîne de la reconnaissance inter-nationale. Mais ce SujetNation ne s'inscrit d'aucune manière dans le nom comme nom de l'élection; 
il ne se remarque, au contraire, que dans la finitude de son nomà l'égard d'un autre nom. En cela, on ne peut juger la scène poli- tique, juridique et constitutionnelle de l'État-Nation moderne comme étant condamnée irrémédiablement au délire des différentes Philosophies de l'Histoire comme procès totalisateur du sens et du nom. On peut y reconnaître, au contraire, le lieu où s'inscrit le nom d'une différence telle qu'en elle-même elle s'articule, de manière critique, au protocole de lecture et d'écriture du politique comme gestion du différend, c'est-à-dire dans la reconnaissance de l'hétérogénéité des jeux de langage qui façonne notre univers politique (Lyotard 1983). Ce désir de reconnaissance du Sujet-Nation québécois peut ainsi s'inscrire également, et par-delà une certaine doxa de la critique de la métaphysique du sujet, dans ce que P. Ricoeur a nommé l'identité narrative; soit, le processus de configuration du sujet dans et par les discours sur l'histoire [14]. Dans ces conditions, le désir de cette signature constitutionnelle n'actualiserait pas, ipso facto, le fantasme du texte national et l'idéal communautaire qu'il implique; au contraire, il me semble qu'il en accomplirait enfin le deuil en s'affranchissant d'une posture fétichiste quant à l'objet (au nom) manquant et au déni de la castration sans laquelle le sujet ne parvient pas à élaborer son désir dans la loi de la filiation du nom du père comme séparation avec la mère. C'est dire en cela également que l'écriture pourrait peut-être, à travers ce travail de deuil, s'ouvrir à une autre élaboration de son désir comme mise en oeuvre d'une déterritorialisation radicale du nom infini de la fondation et faire, enfin, l'épreuve de l'étranger. Le désir de cette signature constitutionnelle du sujet québécois n'est donc pas la mise en place d'un fétiche en lequel se garderait intact le nom de la fondation, mais la réinscription de son nom dans le régime de l'oblitération où se donne à lire, dans sa discernabilité, la finitude du nom comme désir et coupure. Il ne s'agit donc pas, ultimement, de penser cette signature comme le procès d'une adéquation entre le Sujet-Nation et le nom propre, mais du désir du nom du sujet québécois en tant qu'il s'avère enfin lisible dans la différence, c'est-à-dire en tant qu'il puisse se donner à (se) désirer aussi comme indécidable quant au nom qu'il signe. Ce nom propre n'est ainsi que la mise en oeuvre imaginaire de sa discernabilité qu'il joue et risque pour enfin désadhérer du nom infracturable du symbolique. Et c'est à partir de là, sans doute, qu'une critique pertinente de la politique de l'État moderne, et de l'État québécois en particulier, pourrait aussi être posée.

Certes, le texte aquinien se présente selon certains comme un texte éminemment postmoderne, sinon post-colonial, en ce qu'il se donne à lire, formellement, dans cette rhétorique néo-baroque où se déconstruit le procès de la lisibilité du réalisme moderne[15]. Mais il me semble que ce texte ne s'affirme pas comme un texte ironique et parodique quant au désir de réécrire cette histoire en jouant de la citation des formes et des genres. Ce que l'on remarque, au contraire, c'est un baroque malheureux, nostalgique d'un phrasé parfaitement maîtrisé sur la scène de l'histoire. Ce n'est donc pas l'euphorie de l'imaginaire qui est à l'oeuvre ici comme jeu de la coupure du nom et d'un certain règne du simulacre généralisé, mais plutôt la dysphorie imaginaire du sujet à l'égard de l'illisibilité de son nom. Or, l'expérience du texte aquinien amène peut-être à penser que les conditions de possibilité, pour le sujet québécois, d'une expérience authentique de 
cette euphorie sont compromises, sinon difficiles, en raison de cette structure que j'ai dite être celle de l'oblitération et de la double imposture de son nom. Car, en définitive, ce ratage symbolique fait en sorte qu'il est toujours déjà dans cet imaginaire où, semble-t-il, il flotte comme dans un état d'apesanteur historique. Il ne suffirait sans doute qu'un pas pour nommer cette condition l'idéal de la condition postmoderne; pour reconnaître, dans ce sujet québécois, l'idéal de l'imposture et de la déterritorialisation imaginaire. En cela, le sujet québécois ne se nommerait peut-être encore que dans une autre allégorie messianique où, précisément, le délire de cette imposture se donnerait comme un moment de vérité de la culture occidentale, comme elle se donnait, autrefois, comme flambeau de la culture en Amérique (Beaudoin 1989). Or, cette disposition au messianisme n'est-elle pas, tout compte fait, que le symptôme de ce ratage symbolique qui nous donne à fantasmer soi-même et l'autre sur le mode du nom du nom, l'Autre, c'est-à-dire du nom infini de la fondation? Ce serait peut-être là aussi la dernière forme d'une certaine disposition séculaire à l'universalisme abstrait.

À l'heure où le discours de l'identité nationale est positivement remis en question par une nouvelle dynamique basée sur la reconnaissance de la mosaïque culturelle, il est peut-être bon de se rappeler que cette hétérogénéité n'abolit pas, malgré tout, la loi de la discernabilité du nom comme désir. La chance, pourrait-on dire, d'une culture hétérogène au Québec réside aussi dans le désir de cette discernabilité.

"Je dois maintenant, dit Kierkegaard, à la fois être et ne pas être". L'exergue de Neige Noire rappelle sans doute un peu que le désir du nom du sujet québécois ne peut justement se donner à penser, et se donner à désirer, que sur ce double mode de l'identité et de la différence, c'est-à-dire sur le double mode d'un nom enfin reconnu dans son oblitération et qui s'ouvre au désir d'un autre nom reconnu. Comme une signature dont le nom ne se dévoile à l'autre que pour ouvrir une question: indécidable quant à sa finalité historique, mais désirée.

\section{Jacques Cardinal}

\section{Département de littérature comparée}

\section{Université de Montréal}


Surface Page d'Acceuil/Home Page

RÉFÉRENCES BIBLIOGRAPHIQUES

Aquin, Hubert. Prochain Épisode. Montréal: Cercle du Livre de France, 1965.

. Trou de mémoire. Montréal: Cercle du Livre de France, 1968.

. Neige Noire. Montréal: La Presse, coll. Écrivains des deux mondes, 1974.

Beaudoin, Réjean. Naissance d'une littérature. Essai sur le messianisme et les débuts de la littérature canadienne-française (1850-1890). Montréal: Boréal, 1989.

Bilodeau, Rosario, R. Comeau, A. Gosselin et D. Julien. Histoire des Canadas. Montréal: Hurtibise HMH, 1975.

Derrida, Jacques. De la grammatologie. Paris: Minuit, coll. Critique, 1967.

. Otobiographies. L'enseignement de Nietzsche et la politique du nom propre. Paris: Galilée, 1984.

. La faculté de juger. Paris: Minuit, coll. Critique (Colloque de Cerisy, 1982), 1985.

Dubois, Claude-Gilbert. Le Baroque. Profondeurs de l'apparence. Coll Thèmes et textes. Paris: Larousse-Université, 1973.

Fanon, F. Les damnés de la terre. Paris: Maspero, coll. Cahiers Libres, 1961.

Ferron, Jacques. "Une dizaine de petits innocents." In Escarmouches. La longue passe. T.1. Montréal: Leméac, 1975.

Grant, George. Technology and Empire. Perspectives on North America. Toronto: Anansi, 1969. 
Habermas, Jürgen. "La raison communicationnelle: une autre voie pour sortir de la philosophie du sujet." In Le discours philosophique de la modernité (Douze conférences). trad. C. Bouchindhomme et R. Rochlitz. Paris: NRF, Gallimard, coll. Bibliothèque de philosophie, 1988.

Hassan, Ihab. "Pluralism in Postmodern Perspective." Critical Inquiry 12 (Spring 1986).

Lacan, Jacques. Écrits. Paris: Seuil/ Le champ freudien, 1966.

Lacoue-Labarthe, Philippe. L'imitation des modernes (Typographies II). Paris: Galilée, coll. La philosophie en effet, 1986.

Larose, Jean. "Le cheval du réel." Québec studies 9 (1989-90). Article sur le film de Jacques Godbout, Alias Will James, ONFC, 1988.

Larose, Jean. Le mythe de Nelligan. Montréal: Quinze, coll. Prose exacte, 1981.

. La petite noirceur. Montréal: Boréal, coll. Papiers collés, 1987.

Lyotard, Jean-François. Le différend. Paris: Minuit, coll. Critique, 1983.

Major, René. Le discernement. La psychanalyse aux frontières du droit, de la biologie et de la philosophie. Paris: Aubier, coll. La psychanalyse prise au mot, 1984.

. De l'élection. Freud face aux idéologies américaine, allemande et soviétique. Paris: Aubier, coll. La psychanalyse prise au mot, 1986.

Paterson, Janet M. Moments postmodernes dans le roman québéquois.

Ottawa: Presses de l'Université d'Ottawa, 1990.

Ricoeur, Paul. Le temps raconté. Tome 3 de Temps et récit. Paris: Seuil, coll. Essais/Points, 1985: 442-44.

Tapié, Victor L. Baroque et Classicisme. Préface de M. Fumaroli. Coll. Livre de poche. Paris: Pluriel, 1980. 
[1]Cette inscription de la Loi comme coupure et condition de la parole, J. Derrida l'a également fort bien analysée dans son texte sur Kafka, "Préjugés. Devant la Loi":

Devant la Loi, l'homme est sujet de la loi, comparaissant devant elle. Certes. Mais, devant elle parce qu'il ne peut y entrer, il est aussi hors la loi. Il n'est pas toujours sous la loi ou hors la loi. Sujet de la loi: hors la loi. L'homme s'est baissé pour voir à l'intérieur, ce qui laisse entendre que pour l'instant il est plus grand que la porte ouverte, et cette question de la taille nous attend encore. Après qu'il a observé attentivement le gardien, il se décide donc à attendre une permission à la fois donnée et différée mais dont le premier gardien lui laisse anticiper qu'elle sera indéfiniment différée. Derrière le premier gardien il y en a d'autres, en nombre indéterminé; peut-être sont-ils innombrables, de plus en plus puissants, donc de plus en plus interdicteurs, forts de pouvoir différer. Leurs puissances est la différance, une différance interminable puisqu'elle dure des jours, des "années" et finalement jusqu'à la fin de l'homme. Différance jusqu'à la mort, pour la mort, sans fin parce que finie. Représenté par le gardien, le discours de la loi ne dit pas "non" mais "pas encore", indéfiniment. D'où l'engagement dans un récit à la fois parfaitement fini et brutalement interrompu, on pourrait dire primitivement interrompu.

Ce qui est retardé, ce n'est pas telle ou telle expérience, l'accès à une jouissance, à quelque bien fût-il souverain, la possession ou la pénétration de quelque chose ou de quelqu'un. Ce qui est à jamais différé, jusqu'à la mort, c'est l'entrée dans la loi elle-même, qui n'est rien d'autre que cela même qui dicte le retard. La loi interdit en interférant et en différant la "férance", le rapport, la relation, la référence. L'origine de la différance, voilà ce qu'il ne faut pas et ne se peut pas approcher, se présenter, se représenter et surtout pénétrer. Voilà la loi de la loi, le procès d'une loi au sujet de laquelle on ne peut jamais dire "la voilà", ici ou là. Et elle n'est ni naturelle, ni institutionnelle. On y arrive jamais et, au fond de son avoir-lieu originel et propre, elle n'arrive jamais.

(Derrida 1985, 122)

[2]Cette fonction discernante du nom propre, René Major l'a également analysée:

Déployons ces dernières formulations car elles pourront servir de point de départ pour examiner des questions qui transitent à la frontière du droit et de la psychanalyse, des questions qui interrogent le rapport entre le lien analytique et le lien social et ordonnent une autre conception de "la communauté". 
Du nom qui nomme l'innommable, on saisit aisément trois ordres de propositions: 1) le nom fait lien mais $c^{\prime}$ est l'imaginaire qui fait tenir entre elles les propriétés réalistes, semblables ou dissemblables, qui s'y rapportent; 2) la nomination discerne dans la liaison fantasmatique ce qui distingue l'Un des semblables qui feraient tout Un; 3) l'innommable désigne le lieu où le sujet ne renonce à être ni l'Autre ni rien.

Que le sujet se résolve à se prendre et à se déprendre du point de rencontre où ces noms se lient et se délient ne va pas sans une certaine inquiétude dont l'envers est la sérénité qui s'abîme en quelque signifiant suprême. L'innommable, en ce cas, ne saurait être nommé de quelque nom.[...] À toute idée de faire tenir ensemble, et de toute nécessité, des êtres dits semblables au nom du nom qui ne nomme rien et légitime tout, s'opposera l'effet qui nomme le Un comme détaché de l'ensemble, c'est-à-dire à la fois dans la négation de l'ensemble qui le fonde comme Un et dans l'affirmation de l'ensemble qui marque sa distinction. Car, ou l'ensemble assujetti situe dans un for externe le répondant antithétique de son discours, ou le réel dont il s'agit dans le répondant se situe dans ce qui se tient entre et s'en entretient dans l'intervalle de la parole où ce qui se répond ne se correspond pas en se répondant.

(Major 1984, 55-56).

[3]Commentant le processus communautaire de la psychanalyse et de l'État moderne, R. Major écrit:

Entre l'imagination et la raison, dans l'intervalle qui en interrompt et en étoffe la continuité, le Réel comme impossible franchit une double frontière. La marque, valant pour un seul et pour la multiplicité, tenant son identité du rapport de différence qu'elle instaure, de ce qui fait trait et retrait du contexte juridique ou politique, dans la singularité et l'indétermination de son lien, la marque, donc, comme insignifiance marquante, fraye le passage de la limite que découpent les champs du savoir: elle inscrit en même temps, là et ailleurs, la trace d'un écart et la trace du franchissement de cet écart. Ce rapport entretenu de la différence n'exclut ni l'unique ni le différent. Rien n'interdit de trouver là quelques indications concernant les révisions nécessaires pour en finir avec la dissociation du psychanalytique et du juridique, du sujet de l'inconscient et du sujet moral, $c^{\prime}$ est dire pour amorcer une réflexion sur la dignité (au sens kantien) qui soit au-delà de tous les calculs de juridiction.

Par le recours au nom propre, on voit comment le collectif peut faire du trait singulier un trait universel comme trait de la différence et partant, comment l'identification entre eux de ceux qui composent un groupe, voire la multitude, peut se passer de liens libidinaux ou idéologiques et de l'amour d'un chef. 
[4]Or l'expérience du narrateur de Prochain épisode est celle de la dépossession:

Rien n'est libre ici: ni mon coup d'âme, ni la traction adipeuse de l'encre sur l'imaginaire, ni les mouvements pressentis de H. de Heutz, ni la liberté qui m'est dévolue de le tuer au bon moment. Rien n'est libre ici, rien: même pas cette évasion fougueuse que je téléguide du bout des doigts et que je crois conduire quand elle m'efface. Rien! Pas même l'intrigue, ni l'ordre d'allumage de mes souvenirs, ni la mise au tombeau de mes nuits d'amour, ni le déhanchement galiléen de mes femmes. Quelque chose me dit qu'un modèle antérieur plonge mon improvisation dans une forme atavique et qu'une alluvion ancienne étreint le fleuve instantané qui m'échappe. Je n'écris pas, je suis écrit.

(Aquin 1965, 89-90)

Quand il a commencé son baratin, il savait déjà que je ne pouvais pas tomber dans une trappe aussi grossière. Il a sûrement prévu que je ne serais pas dupe de son stratagème incroyable. Dans ce cas, s'il a brodé sur le schéma que j'ai moi-même développé ce matin, ce n'est pas par accident, ni par une combinaison fortuite due aux simples lois de la probabilité. H. de Heutz a donc obéi à un plan précis. Il avait une idée derrière la tête en m'entraînant dans cette charge d'invraisemblance et d'ironie. Laquelle? Peut-être a-t-il voulu me transmettre un message chiffré. Mais non, je déraisonne puisque entre $H$. de Heutz et moi il ne saurait y avoir de chiffre, ni de code, ni aucune raison d'échanger quelque message que ce soit. C'est la rupture implacable et l'impossibilité de communiquer autrement que sous forme de coups de feu. Si j'en suis rendu à analyser les intentions profondes de son comportement avec moi, peut-être, au fond, suis-je sur le point de tomber dans le piège qu'il m'a tendu, et que je réagis très exactement comme il l'a voulu? Ma fascination même ainsi que son corollaire de doute méthodique et d'hésitation --, il l'a provoquée sciemment. Mais pourquoi?

Cet inconnu que je regarde m'attire à l'instant même où je m'apprête à le tuer. Son mystère déconcerte ma préméditation et je reste pantelant devant lui, incapable de diriger mes pensées vers un autre objet et de combattre l'attirance morbide qu'il exerce sur moi. [...] L'agilité supersonique de mon esprit s'affaisse soudainement sous le charme maléfique de H. de Heutz. Je m'immobilise, métamorphosé en statue de sel, et ne puis m'empêcher de me percevoir comme foudroyé.[...] Je continue de le regarder, j'entends ces sanglots, et une sorte de mystère me frappe d'une indécision sacrée. Un 
événement que j'ai cessé de contrôler s'accomplit solennellement en moi et me plonge dans une transe profonde. (Aquin 1965, 117-121)

$[6]$

A la place du nom du propriétaire, se trouve un dessin chargé qui s'enroule sur lui-même dans une série de boucles et de spires qui forment un noeud gordien, véritable agglomérat de plusieurs initiales surimprimées les unes sur les autres et selon tous les agencements graphiques possibles.

Il en est ainsi du château tout entier qui me mystifie non pas tellement en tant qu'habitacle, mais en tant que chiffre. Car ces coffres ciselés qui ne contiennent rien, ces médaillons qui reflètent des images de guerre et ce livre apparemment oublié qui raconte les guerres de César, voilà autant d'initiales nouées inextricablement dans un fouillis hautain et fascinant. Tout cela porte une signature, celle de l'homme que j'attends.

(Aquin 1965, 130-131).

On peut aussi lire le cryptogramme comme le nom illisible de cette capture imaginaire au champ de l'Autre:

En butant sur cette équation à multiples inconnues que je dois résoudre avant d'aller plus loin dans mon récit, j'ai le sentiment de me trouver devant le mystère impénétrable par excellence. Plus je le cerne et le crible, plus il croît au-delà de mon étreinte, décuplant ma propre énigme lors même que je multiplie les efforts pour le saisir. Je n'arrive pas à réinventer le code de ce message; et faute de le traduire dans mon langage, j'écris dans l'espoir insensé qu'à force de paraphraser l'innommable, je finirai par le nommer.

(Aquin 1965, 21-22)

[7] Pour une analyse détaillée de cette inscription, je réfère le lecteur à mon livre, Le roman de l'histoire. Politique et transmission du nom dans Prochain épisode et Trou de mémoire d'Hubert Aquin (Montréal: Balzac, coll.

L'univers des discours. À paraître en 1993.

[87Cette parataxe est d'abord lisible formellement dans ce chiasme narratif et diégétique entre le présent d'une incarcération psychiatrique et le temps de l'imaginaire romanesque. Elle l'est également comme le noeud d'une inscription incertaine du sujet quant à la reconnaissance de son identité comme mise en oeuvre de la lisibilité de son nom et de son histoire: 
Ce livre défait me ressemble. Cet amas de feuilles est un produit de l'histoire, fragment inachevé de ce que je suis moi-même et témoignage impur, par conséquent, de la révolution chancelante que je continue d'exprimer, à ma façon, par mon délire institutionnel.

(Aquin 1965, 92)

Or le dénouement de cette histoire est, en définitive, une promesse:

Mon récit est interrompu, parce que je ne connais pas le premier mot du prochain épisode. Mais tout se résoudra en beauté. J'ai confiance aveuglément, même si je ne connais rien du chapitre suivant, mais rien, sinon qu'il m'attend et qu'il m'emportera comme un tourbillon. Tous les mots de la suite me prendront à la gorge; l'antique sérénité de notre langue éclatera sous le choc du récit. Oui, l'invariance de ce qui se raconte subira la terreur impie; des sigles révolutionnaires seront peinturés au fusil à longueur de pages. [...] Non, je ne finirai pas ce livre inédit: le dernier chapitre manque qui ne me laissera même pas le temps de l'écrire quand il surviendra. Ce jour-là, je n'aurai pas à prendre les minutes du temps perdu. Les pages s'écriront d'elles-mêmes à la mitraillette: les mots siffleront audessus de nos têtes, les phrases se fracasseront dans l'air. [...] Oui, je sortirai vainqueur de mon intrigue, tuant $H$. de Heutz avec placidité pour me précipiter vers toi, mon amour, et clore mon récit par une apothéose.

(Aquin 1965, 171-73)

[9]Ainsi que se termine le roman:

Depuis, tant de choses se sont passées: j'ai changé de nom, je porte un enfant qui s'appellera Magnant - et jusqu'au bout, je l'espère, et sans avoir peur de son nom. Et je veux que mon enfant soit plus heureux que son père et qu'il n'apprenne jamais comment il a été conçu, ni mon ancien nom...

(Aquin 1968, 204)

[10]L'on sait pertinemment que le tableau d'Holbein, Les Ambassadeurs, est le paradigme du roman. En lui se donne à lire les deux temps constitutifs de la représentation du sujet en ce qu'il montre d'abord, dans une esthétique de la discernabilité ou de l'imitation, les insignes du pouvoir et de la maîtrise de l'homme sur le monde et, d'autre part, le refoulé mortifère de cette maîtrise qui est enkysté dans le tissu de cette représentation, et selon une autre construction géométrique, qui est celle de l'anamorphose. 
[11]Encore une fois, on constate que la poétique à l'oeuvre dans ce texte est celle d'une certaine parataxe, nostalgique, en définitive, d'une phraséologie syntaxico-linéaire capable d'assurer la reconnaissance du sujet dans l'Histoire:

Mais justement, ce pays n'a rien dit, ni rien écrit: il n'a pas produit de conte de fée, ni d'épopée pour figurer, par tous les artifices de l'invention, son fameux destin de conquis: mon pays reste et demeurera longtemps dans l'infra-littérature et dans la sous-histoire. C'est tout juste s'il enfante quelques malades comme moi, de ci de là, en pur gaspillage et sans les nommer... Les fabricants d'histoire ne savent plus où donner de la tête: ils s'en vont dans la vie, avec quelques bonnes répliques, mais il n'y a pas de contexte, ni même de sous-textes dans lesquels ils pourraient insérer leurs périodes. Alors, ils restent là, debout, avec leurs apocopes à la main, hébétés, plantés comme des cocus dans une intrigue muette qui, fertile en sous-entendus, n'est finalement entendue par personne! [...] Le Québec, c'est cette poignée de comédiens bègues et amnésiques qui se regardent et s'interrogent du regard et qui semblent hantés par la platitude comme Hamlet par le spectre.

(Aquin 1968, 55)

Cette violence fondatrice comme cérémonial de lustration est, en définitive, la suture de l'obscénité de la violence. Ainsi les séances de narco-analyses de Olympe Ghezzo-Quenum aux dépends de Rachel Ruskin ont-elles pour buts de lui faire avouer sa jouissance dans le viol (170-71).

[12]Rappellons ici la position de F. Fanon à l'égard de la violence révolutionnaire en situation coloniale:

La mise en question du monde colonial par le colonisé n'est pas une confrontation rationnelle des points de vue. Elle n'est pas un discours sur l'universel, mais l'affirmation échevelée d'une originalité posée comme absolue. (47)

Le colonialisme n'est pas une machine à penser, n'est pas un corps doué de raison. Il est la violence à l'état de nature et ne peut s'incliner que devant une plus grande violence. (64)

La violence est ainsi comprise comme la médiation royale. L'Homme colonisé se libère dans et par la violence. [...] Au niveau des individus, la violence désintoxique. (71) 
[13]Pour un historique de cette opposition et de son usage idéologique, je refère le lecteur à la préface écrite par Marc Fumaroli au livre de Victor L. Tapié, Baroque et classicisme (Paris: Livre de poche, coll. Pluriel, 1980). De même, le livre de Claude-Gilbert Dubois, Le baroque. Profondeurs de l'apparence (Paris: Larousse-Université, coll. Thèmes et textes, 1973).

\section{$[14]$}

Le rejeton fragile issu de l'union de l'histoire et de la fiction, c'est l'assignation à un individu ou à une communauté d'une identité spécifique qu'on peut appeler leur identité narrative. "Identité" est pris ici au sens de catégorie de la pratique. Dire l'identité d'un individu ou d'une communauté, c'est répondre à la question: qui a fait telle action? qui est l'agent, l'auteur? Il est d'abord répondu à cette question en nommant quelqu'un, c'est-à-dire en le désignant par un nom propre. Mais quel est le support de la permanence du nom propre? Qu'est-ce qui justifie qu'on tienne le sujet de l'action, ainsi désigné par son nom, pour le même tout au long d'une vie qui s'étire de la naissance à la mort? La réponse ne peut être que narrative. Répondre à la question "qui?", comme l'avait fortement dit Hannah Arendt, c'est raconter l'histoire d'une vie. L'histoire racontée dit le qui de l'action. L'identité du qui n'est donc elle-même qu'une identité narrative. Sans le secours de la narration, le problème de l'identité personnelle est en effet voué à une antinomie sans solution: ou bien l'on pose un sujet identique à lui-même dans la diversité de ses états, ou bien l'on tient, à la suite de Hume et de Nietzsche, que ce sujet identique n'est qu'une illusion substantialiste, dont l'élimination ne laisse apparaître qu'un pur divers de cognitions, d'émotions, de volitions. Le dilemme disparaît si, à l'identité comprise au sens d'un même (idem), on substitue l'identité comprise au sens d'un soimême (ipse); la différence entre idem et ipse n'est autre que la différence entre une identité substantielle ou formelle et l'identité narrative. L'ipséité peut échapper au dilemme du Même et de l'Autre, dans la mesure où son identité repose sur une structure temporelle conforme au modèle d'entité dynamique issue de la composition poétique d'un texte narratif. Le soi-même peut ainsi être refiguré par l'application réflexive des configurations narratives. À la différence de l'identité abstraite du Même, l'identité narrative, constitutive de l'ipséité, peut inclure le changement, la mutabilité, dans la cohésion d'une vie. Le sujet apparaît alors constitué à la fois comme lecteur et comme scripteur de sa propre vie, selon le voeu de Proust. [...] On peut parler de l'ipséité d'une communauté, comme on vient de parler de celle d'un sujet individuel: individu et communauté se constituent dans leur identité en recevant tels récits qui deviennent pour l'un comme pour l'autre leur histoire effective.

(Ricoeur 42-44)

[15]Ce jugement, on peut le lire notamment dans le discours critique de Janet M. Paterson dans son livre Moments postmodernes dans le roman québécois (Ottawa: Les presses de l'université d'Ottawa, 1990). 
Formellement, l'analyse est pertinente; mais elle laisse intacte à mon avis la question de la symbolisation. 